\title{
IMPURITY INDUCED DISORDERING PRODUCED LATERAL OPTICAL CONFINEMENT IN AlGaAs AND InGaAs (ON GaAs) QUANTUM WELL WAVEGUIDES
}

\author{
Alex T. H. Li, K. M. Lo and E. Herbert Li \\ Department of Electrical and Electronic Engineering \\ The University of Hong Kong, Pokfulam Road, Hong Kong
}

\begin{abstract}
Impurity induced disordering technique is employed to an AlGaAs/GaAs quantum well optical waveguide to provide lateral optical confinement. The modal propagation constant and field profile are analysed using an improved Fourier decomposition method. Single mode operating region are given in terms of thickness of quantum well layers.
\end{abstract}

\section{INTRODUCTION}

The control modification of the rate of $\mathrm{Al} / \mathrm{Ga}$ interdiffusion, such as by the introduction of impurities on masked pattern, interdiffusion across $\mathrm{Al}_{\mathrm{w}} \mathrm{Ga}_{1-\mathrm{w}} \mathrm{As} / \mathrm{GaAs}$ quantum well (QW) interface is a process of considerable interest for the fabrication of optoelectronic device [1]. This impurity induced disordering (IID) technique provides an efficient way to realize two dimensional (2-D) waveguiding structure in optoelectronic integrated circuits. The electronic and optical properties of the interdiffusion modified AlGaAs/GaAs QW strucures, such as the band strucutre and refractive index have been studied [2], [3]. A 2$D$ waveguide structure fabricated by using the IID technique has been studied theoretically and a simple model is used to describe the interdiffusion profile and band-gap shifts [4], however, the modal properties of waveguides have not been considered. In this paper, a detailed model of the $2-\mathrm{D}$ waveguide structure fabricated using such technique is presented. In the next section, an outline is given in the implementation of a 2-D waveguide using the IID technique. In Section III, a numerical method is employed to solve the wave equation. Solution of the equation are modal propagation constant and field amplitude. Results are presented in Section IV. Finally, the requirement for single mode operation is presented.

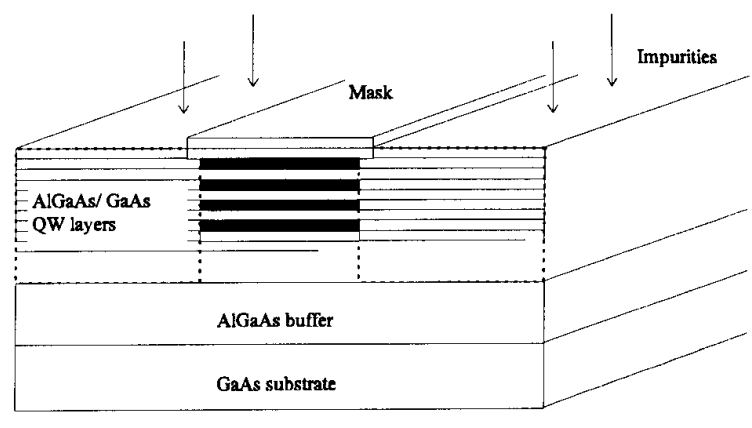

Fig. 1. The schematic of cross section of IID QW waveguide

\section{IMPLEMENTATION}

The structure to be modeled consists of layers of GaAs QW on a thick $\mathrm{Al}_{0.3} \mathrm{Ga}_{0.7}$ As buffer layer. The schematic of the structure is show in Fig. 1. The implantation process produces a modification of the $\mathrm{QW}$ material which in turn leads to differences in refractive index in different region [5]. The implanted region has lower refractive index than the non-implanted region, hence produce lateral confinement for light and a 2$\mathrm{D}$ waveguide is formed. The refractive index of the waveguiding strucutre fabricated by such technique is inhomogeneous.

The steps involved in the implementation of IID QW waveguide is illustrated in Fig. 2. To find out the refractive index profile of $\mathrm{QW}$ layers, we computed the impurity density as implanted for a $2-\mathrm{D}$ ( $x-y$ axes) structure, where $x$ is defined as the lateral direction of the waveguide and the depth $y$ is perpendicular to it. The concentration of the implanted impurity is described by an equation base on the experimental and simulation results [6], such as the one shown as follows:

$$
N(x, y)=\frac{N_{o}}{\sqrt{2 \pi} \Delta R_{p}} \exp \left[-\frac{\left(y-R_{p}\right)^{2}}{2 \Delta R_{p}^{2}}\right]
$$




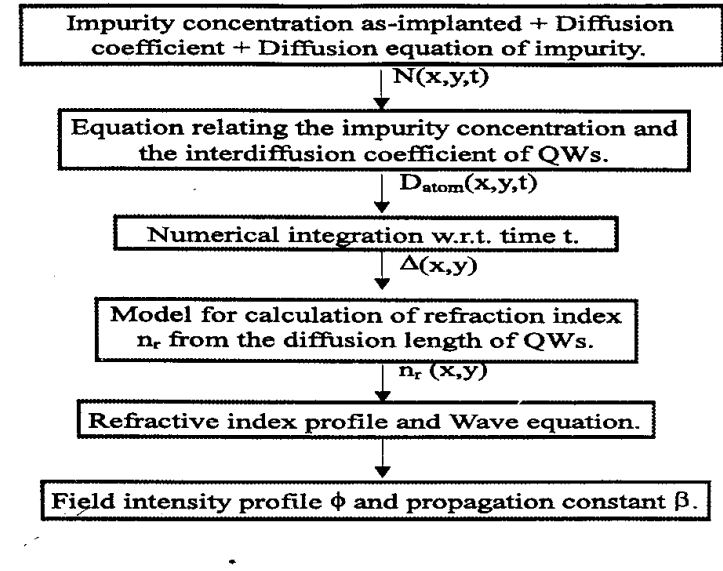

Fig. 2. Flow chart to describe steps for implementation.

$$
\begin{aligned}
& \times \frac{1}{2}\left[\operatorname{erfc}\left(\frac{x-a}{\sqrt{2 \Delta R_{p L}}}\right)\right. \\
& \left.-\operatorname{erfc}\left(\frac{x+a}{\sqrt{2 \Delta R_{p L}}}\right)\right]
\end{aligned}
$$

where $N_{o}$ is the implanted dose, $2 a$ is the mask width, $\Delta R_{p}$ is the standard derivation of the projected range $R_{p}$, and $\Delta R_{p L}$ is the lateral spread.

When the sample was heated, diffusion of $\mathrm{Al} / \mathrm{Ga}$ and the defect occurs simultaneously. Their motion is described by a classical diffusion law. For short times we shall assume that the diffusion of the impurity is independent of the motion of $\mathrm{Al} / \mathrm{Ga}$ atoms and the diffusion coefficients $D_{\text {ion }}$ is a constant through out the sample for any time $t$. The diffusion equation for the impurities is

$$
\frac{\partial N(x, y, t)}{\partial t}=D_{\mathrm{ion}} \nabla^{2} N(x, y, t)
$$

where $N(x, y, t)$ is the impurity concentration profile.

We shall also assume the interdiffusion coefficient, $D_{\text {atom }}$, depends on the local defect density only which is described by the equation, $D_{\text {atom }}(x, t)=$ $\alpha D_{\text {ion }} N(x, y, t)$, where $\alpha$ is a numerical factor determined from experimental data. The diffusion equation for the $\mathrm{Al} / \mathrm{Ga}$ atoms is

$$
\frac{\partial C(x, y, t)}{\partial t}=\nabla \cdot\left[D_{\text {atom }}(x, y, t) \nabla C(x, y, t)\right]
$$

where $C(x, y, t)$ is the $\mathrm{Al} / \mathrm{Ga}$ atom concentration.

Once we have obtain the $\mathrm{Al} / \mathrm{Ga}$ concentration profile, the position dependent diffusion length of the $\mathrm{Al} / \mathrm{Ga}$ atoms at any time can be obtained. Using a previously developed model [7], a 2-D refractive index profile $n_{r}(x, y)$ was then calculated from these diffusion lengths, as shown in Fig. 3.

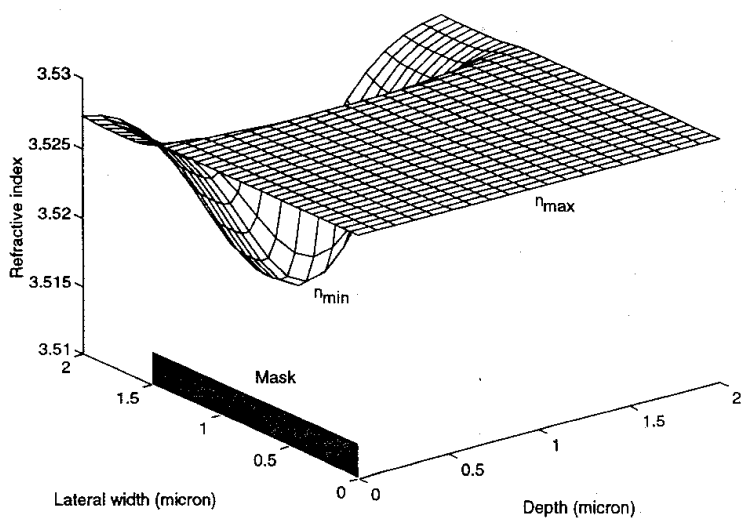

Fig. 3. A typical refractive index profile (half symmetry) of a waveguide structure with 20 multi-QW layers, mask width of $1.5 \mu \mathrm{m}$ and operating wavelength at $0.9 \mu \mathrm{m}$.

\section{WAVE EQUATION}

Consider a translationally invariant, real refractive index profile, $n_{r}(x, y)$, which only changes slightly across the QW layers as shown in Fig. 3. The maximum refractive index of the $\mathrm{QW}$ layers $n_{\max }$ is similar to the minimum refractive index of the $\mathrm{QW}$ layers $n_{\min }$, and weak-guidance approximation is applicable. Within this approximation, all polarization properties of the waveguide are neglected and Maxwell's equations reduce to the scalar wave equation:

$$
\left[\frac{\partial^{2}}{\partial x^{2}}+\frac{\partial^{2}}{\partial y^{2}}+k^{2} n_{r}^{2}(x, y)-\beta^{2}\right] \Psi(x, y)=0
$$

Here, $\Psi(x, y)$ denotes the transverse modal electric field, $k=2 \pi / \lambda$, where $\lambda$ represents the free-space wavelength and $\beta$ is the associated propagation constant. It should be noted that there are no exact analytical solutions of Eq. (4) for the QW waveguide illustrated in Fig. 3. To this end, an improved Fourier decomposition method (FDM), based on the mapping of the whole $x-y$ plane onto a unit square in $u-v$ space was adopted to solve the scalar wave equation [8].

For the below calculations, 30 harmonics (in each direction) in the Fourier series are used to expand the mode field in a mapped space (resulting in a matrix equation of size $900 \times 900$ ).

\section{MODAL PROPERTIES}

For a given refractive index profile, $n_{r}(x, y)$, the normalised propagation constant $P^{2}$ is calculated, where $P^{2}=\left[(\beta / k)-n_{\min }^{2}\right] /\left(n_{\max }^{2}-n_{\min }^{2}\right)$. Fig. 4 shows the normalised propagation constants as a function of $\mathrm{QW}$ layers thickness, $t_{\mathrm{QW}}$. The guided mode of the wave- 


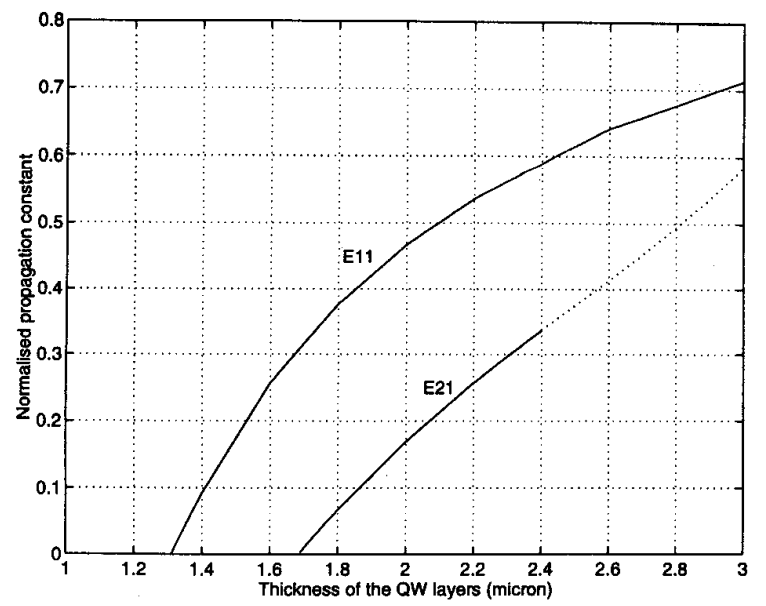

Fig. 4. Normalised propagation constant as a function of thickness of the QW layers with mask width of $1.5 \mu \mathrm{m}$ and operating wavelength at $0.9 \mu m$.

guide is denoted by $E_{\mathrm{mn}}$ mode ( $m, n$ are both positive integers) with $m-1$ and $n-1$ field zeros in the $x$ and $y$ directions, respectively. The symmetric $E_{11}$ field profiles, for the case $t_{\mathrm{QW}}=1.6 \mu \mathrm{m}$ is displayed in Fig. 5 . The corresponding confinement factor or fraction of power in the core $\eta$ is $94.7 \%$. Where

$$
\eta=\frac{\int_{A_{\text {core }}} \Psi(x, y)^{2} \mathrm{dA}}{\int_{A_{\infty}} \Psi(x, y)^{2} \mathrm{dA}}
$$

and $A_{\text {core }}$ is the area of QW layers under the mask.

The antisymmetric $E_{21}$ field profiles, for the case $t_{\mathrm{QW}}=2.2 \mu \mathrm{m}$ is displayed in Fig. 6 .

Finally, the single mode (SM) region is considered. To have single mode operation, the high order mode, $E_{21}$ mode, must be cutoff and only the fundamental mode, $E_{11}$ mode, propagates. Therefore, the thickness of QW layers for SM operation is from $1.3 \mu \mathrm{m}$ to $1.7 \mu \mathrm{m}$.

\section{CONCLUSION}

The modal properties of a multi-quantum well waveguide fabricated using the impurity induced disordering technique is investigated. Modal properties include propagation constant, modal field, confinement factor and single mode operating region. The modal study is based on an improved Fourier decomposition method.

The algorithms developed in present studies can also applied to other optical waveguide employing the impurity induced disordering technique. Example like InGaAs/GaAS quantum well waveguide.

\section{ACKNOWLEDGMENT}

This work is supported by the HKU-CRCG grant.
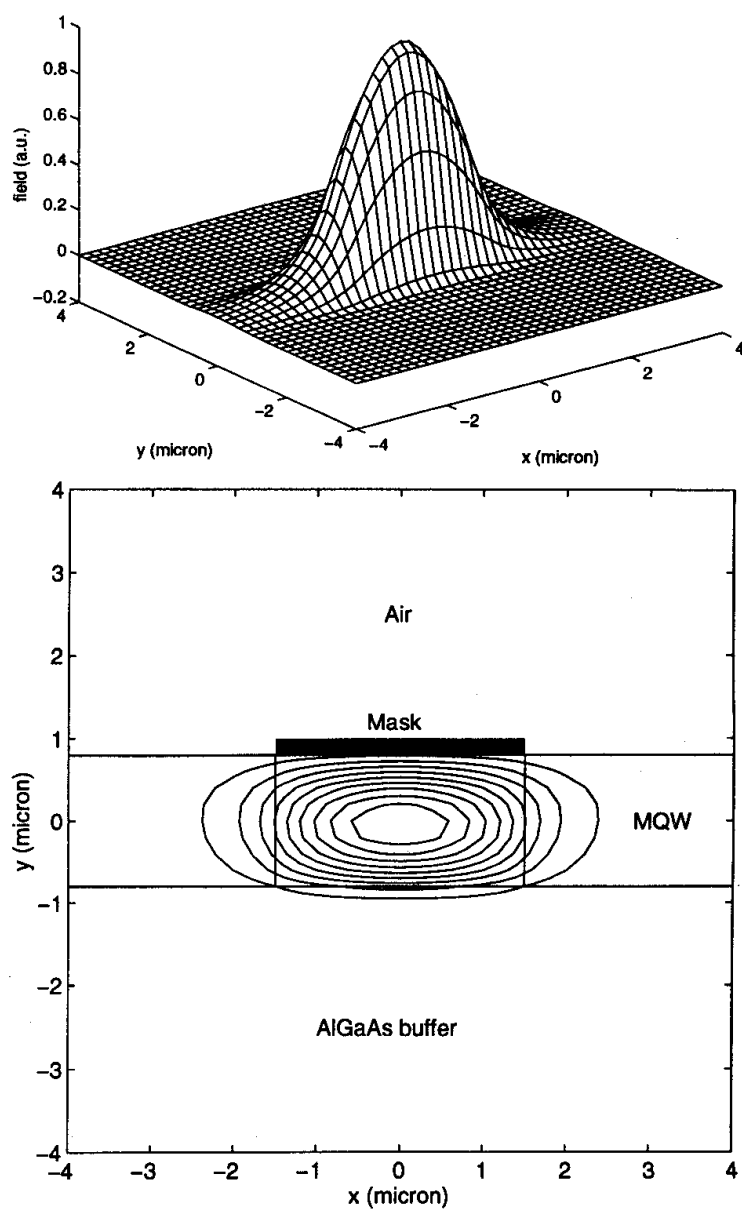

Fig. 5. Symmetric $E_{11}$ field profile as determined by FDM with QW layers thickness of $1.6 \mu \mathrm{m}$. Contour levels are at $10 \%$ intervals of the maximum field amplitude.

\section{REFERENCES}

[1] B. L. Weiss, Ed., Special Issue on Quantum Well Mixing for Optoelectronics, vol. 23 of Opt. Quantum Electron., 1991.

[2] E. H. Li, B. L. Weiss, and K. S. Chan, "Effect of interdiffusion on the subbands in an $\mathrm{Al}_{\mathbf{x}} \mathrm{Ga}_{1-\mathrm{x}} \mathrm{As} / \mathrm{GaAs}$ single quantum-well structure", Phys. Rev. B., vol. 46, pp. 15181-15192, 1992.

[3] E. H. Li and B. L. Weiss, "The change in refractive index of AlGaAs/GaAs single quantum wells due to impurity-induced mixing", IEEE Photon. Technol. Lett., vol. 3, pp. 787-789, 1991.

[4] J. Cibert and P. M. Petroff, "Carrier confinement potential in quantum-well wires fabricated by implantation-enhanced interdiffusion in the $\mathrm{GaAs}-\mathrm{Ga}_{1-\mathrm{x}} \mathrm{Al}_{\mathrm{x}}$ As system", Phys. Rev. B., vol. 36, pp. 3243-3246, 1987. 

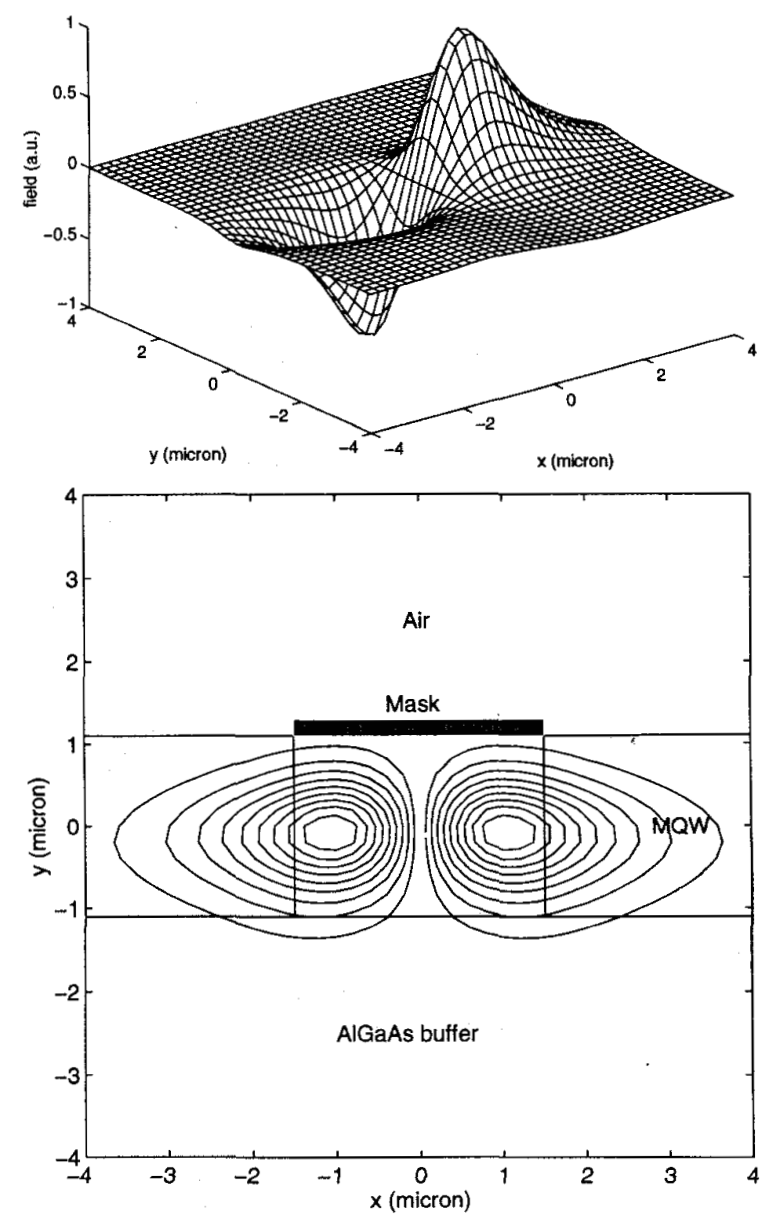

Fig. 6. Antisymmetric $E_{21}$ field profile as determined by FDM with QW layers thickness of $2.2 \mu \mathrm{m}$. Contour levels are at $10 \%$ intervals of the maximum field amplitude.

[5] T. Wolf, C. L. Shieh, R. Engelmann, K. Alavi, and J. Mantz, "Lateral refractive index step in $\mathrm{Ga} / \mathrm{As} / \mathrm{AlGaAs}$ multiple quantum well waveguide fabricated by impurity-induced disordering", Appl. Phys. Lett., vol. 55, pp. 1412-1414, 1989.

[6] D. Briggs and M. P. Seah, Eds., Ion and Neutral Spectroscopy, vol. 2 of Practical Surface Analysis, Wiley, Chichester, 2nd edition, 1992.

[7] E. H. Li, B. L. Weiss, K. S. Chan, and J. Micallef, "Polarization dependent refractive index of an interdiffusion induced AlGaAs/GaAs quantum well", Appl. Phys. Lett., vol. 62, pp. 550-552, 1993.

[8] S. J. Hewlett and F. Ladouceur, "Fourier decom position method applied to mapped infinite domains: scalar analysis of dielectric waveguides down to modal cutoff", J. Lightwave Technol., vol. 13, pp. 375-383, 1995. 